\title{
Primera cita de Cotoneaster pannosus Franch. (Rosaceae) para la provincia de Tarragona y actualización corológica para España
}

\author{
Jordi López-Pujol y Daniel Guillot Ortiz²
}

\begin{abstract}
Resumen: López-Pujol, J. \& Guillot Ortiz, D. 2015. Primera cita de Cotoneaster pannosus Franch. (Rosaceae) para la provincia de Tarragona y actualización corológica para España. Bot. Complut. 39: 63-69.

Se cita por primera vez en la provincia de Tarragona (Cataluña, España) a la especie alóctona Cotoneaster pannosus (Rosaceae), arbusto de uso ornamental originario de China. Se ofrecen datos sobre su demografía y especies acompañantes para la nueva localidad, además de la actualización corológica para España.
\end{abstract}

Palabras clave: flora alóctona, naturalizado, ornamental.

Abstract: López-Pujol, J. \& Guillot Ortiz, D. 2015. First report of Cotoneaster pannosus Franch. (Rosaceae) in Tarragona Province, and updated distribution area in Spain. Bot. Complut. 39: 63-69.

Cotoneaster pannosus (Rosaceae), an ornamental shrub native to China, is reported for the first time in Tarragona Province (Catalonia, Spain). Data on demography and accompanying species for the new locality are provided, in addition to a chrorological update at nation level.

Key words: alien flora, naturalized, ornamental.

\section{INTRODUCCION}

En los últimos años se viene reportando la presencia de numerosos táxones alóctonos introducidos en nuestro país con fines ornamentales (Sanz et al. 2004, 2009; Andreu \& Vilà 2010; Fraga \& Del Toro 2010). Aunque muchas de las rosáceas alóctonas presentes en España se introdujeron como árboles frutales (géneros Malus, Prunus y Pyrus) un buen número de ellas se han asilvestrado como consecuencia de su utilización en jardinería. El género Cotoneaster es ampliamente usado en jardinería debido a su elegante porte y a la belleza de sus flores y frutos. Se trata de un género que podría alcanzar las 400 especies, que se distribuyen por el norte de África, Europa y en numerosas áreas templadas de Asia (Fryer \& Hylmö 2009). Con su centro de diversificación en los Himalayas y las montañas del sudoeste de China (existen alrededor de 200 especies endémicas de China; Fryer \& Hylmö 2009), en España podría haber hasta cuatro especies nativas [(Cotoneaster favargeri J. Fryer \& B. Hylmö, C. granatensis Boiss., C. integerrimus
Medik. y C. tomentosus (Aiton) Lindl. (Blanca 1998)]. En nuestro país se han citado como alóctonos a un mínimo de seis especies del género: Cotoneaster coriaceus Franch. (C. lacteus W. W. Sm.), C. franchetii, C. horizontalis Decne., C. pannosus Franch., C. racemiflorus (Desf.) K. Koch, y C. symondsii T. Moore (Sanz et al. 2004, 2011; Andreu \& Pino 2013; Aymerich 2013b). Cotoneaster pannosus es uno de los menos frecuentes en España; de hecho la primera cita como alóctono no se recoge hasta 1989, y hasta la fecha sólo se había observado su presencia en las provincias de Barcelona, Castellón, Girona y Valencia, contabilizando un máximo de 20 localidades en total (véase Resultados y Discusión para más detalles). Se cita por primera vez como alóctono en la provincia de Tarragona, lo que supone una importante novedad corológica a escala nacional.

\section{MATERIALES Y MÉTODOS}

En el curso de una prospección sobre flora naturalizada en la provincia de Tarragona (NE de España) se descubrió una pe-

\footnotetext{
${ }^{1}$ Institut Botànic de Barcelona (IBB-CSIC-ICUB). Passeig del Migdia s/n, 08038, Barcelona, España, jlopezpu@gmail.com

2 Jardín Botánico, Universidad de Valencia.C/ Quart 82, 46008, Valencia, España, dguillot_36@hotmail.com

Recibido: 21 diciembre 2014. Aceptado: 21 enero 2015.
} 
queña población de Cotoneaster pannosus. La identificación se realizó mediante literatura especializada (Lu \& Brach 2003, Fryer \& Hylmö 2009) y consulta de los herbarios BC y BCN. Se ha depositado pliego testigo en el herbario del Instituto Botánico de Barcelona (BC). Se realizó un censo de los ejemplares, además observar los estadios de desarrollo. Finalmente, se anotaron las especies acompañantes y se tomaron numerosas fotografías (mediante una cámara digital Finepix HS30; Fujifilm, Tokio, Japón).

\section{RESULTADOS Y DISCUSIÓN}

\section{Cotoneaster pannosus Franch.}

T: Tarragona (Cala Romana), 31TCF5654, ca. $20 \mathrm{~m}$, terreno inculto, junto a la carretera N-340, 24-X-2014, López-Pujol, BC 941214.

La población que hemos detectado en la provincia de Tarragona (Fig. 1), primera de esta demarcación, es un pequeño núcleo de unos cuarenta individuos, bien estructurada demográficamente (con individuos jóvenes y adultos; Fig. 2), y que se extiende por un área no superior a 1 ha. La mayoría de individuos producen abundantes frutos (Fig. 2), y algunos de ellos superan los $4 \mathrm{~m}$ de altura. Aunque en la descripción de Flora of China se detalla que la especie alcanza unos 2 metros de altura (Lu \& Brach 2003), algunas observaciones —además de la nuestra - confirman que puede superar los $3 \mathrm{~m}$ e incluso alcanzar los $5 \mathrm{~m}$ (Lorence et al. 1995, Herrero-Borgoñón et al. 2005, Queensland Government 2011), tal y

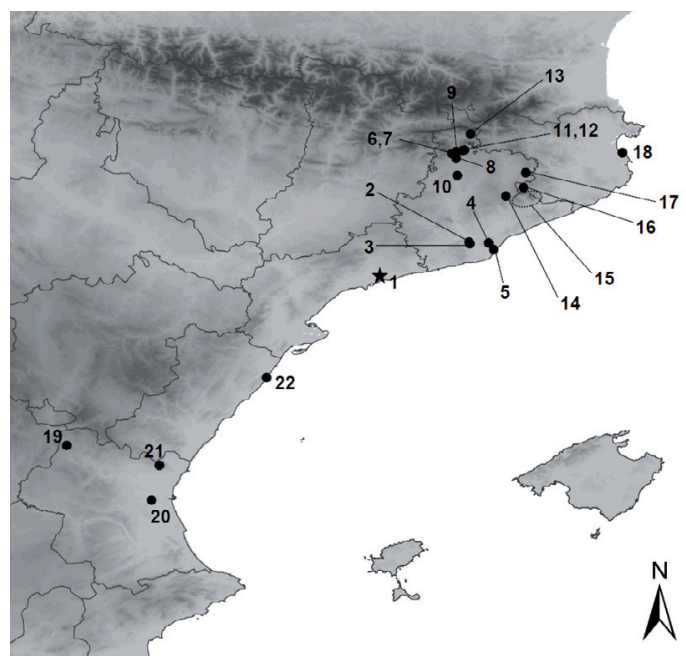

Fig. 1- Localidades donde se ha reportado la presencia de Coroneaster pannosus en España. Los detalles de las localidades se encuentran en la Tabla 1. como también se afirma en la descripción propuesta en la monografía de Fryer \& Hylmö (2009). La población se encuentra bajo un pequeño pinar de Pinus halepensis, con restos de maquia litoral (Asparagus acutifolius, $\mathrm{Ce}$ ratonia siliqua, Chamaerops humilis, Olea europaea var. sylvestris, Pistacia lentiscus, Rhamnus alaternus, $R$. lyciodes, Rubia peregrina y Smilax aspera). Convive con numerosas alóctonas (de las que destacan Agave franzosini, A. ingens var. picta, Agave sp., Albizia julibrissin, Aloe maculata, Asparagus plumosus, Lantana camara, Ligustrum lucidum, Opuntia cf. ficus-indica, Pittosporum tobira y Senecio angulatus). La presencia de estos xenófitos, la mayoría de ellos ampliamente usados en jardinería, junto con Nerium oleander y Coronilla cf. glauca (especies, aunque autóctonas, también usadas como ornamentales), no resulta sorprendente dada la cercanía de una de las principales - y más antiguas - urbanizaciones de casas unifamiliares de la ciudad de Tarragona (Cala Romana).

Cotoneaster pannosus es un arbusto semiperennifolio originario del sudoeste de China (ampliamente distribuido en provincias de Sichuan y Yunnan y sureste de la Región Autónoma del Tíbet, y localmente presente en el oeste de la provincia de Guizhou; véase Fang et al. 2011 y http://www.cvh.org.cn/). Se diferencia de su congénere más cercano presente en España ( $C$. franchetii) por tener un pecíolo más largo, un mayor número de flores por cima, por el número de semillas por pomo (dos en C. pannosus, generalmente tres en $C$. franchetii), y por el color del fruto (generalmente de un rojo más intenso en $C$. pannosus) (Herrero-Borgoñón 2003, Lu \& Brach 2003). Cultivado en muchos lugares con fines ornamentales, se ha naturalizado en Australia (incluyendo la isla de Tasmania; Baker 2005; Queensland Government 2011) y Nueva Zelanda (Heenan et al. 2004), en el África austral (Lesotho, Suráfrica, Swazilandia y Zimbabwe; Macdonald et al. 2013; Hyde et al. 2014; SAPD 2014), en Estados Unidos continental (Oregon y California; Cal-IPC 2006; Anónimo 2014), en Colombia (Camelo et al. 2012), en Uruguay (InBUy 2011), en las islas Hawaii (Lorence et al. 1995), en el archipiélago de Madeira (Vieira 2002), además de en varios países europeos (como mínimo España, Italia, Gran Bretaña, y probablemente en Francia; Celesti-Grapow et al. 2009; DAISIE 2014; NNSS 2014; Valkenburg et al. 2014).

En España se ha reportado su presencia en estado silvestre únicamente en Cataluña (Andreu \& Pino 2013) y la Comunidad Valenciana (Sanz et al. 2011) (Fig. 1), aunque es probable que esté presente en muchas otras comunidades (puesto que existen numerosas citas de su culti- 

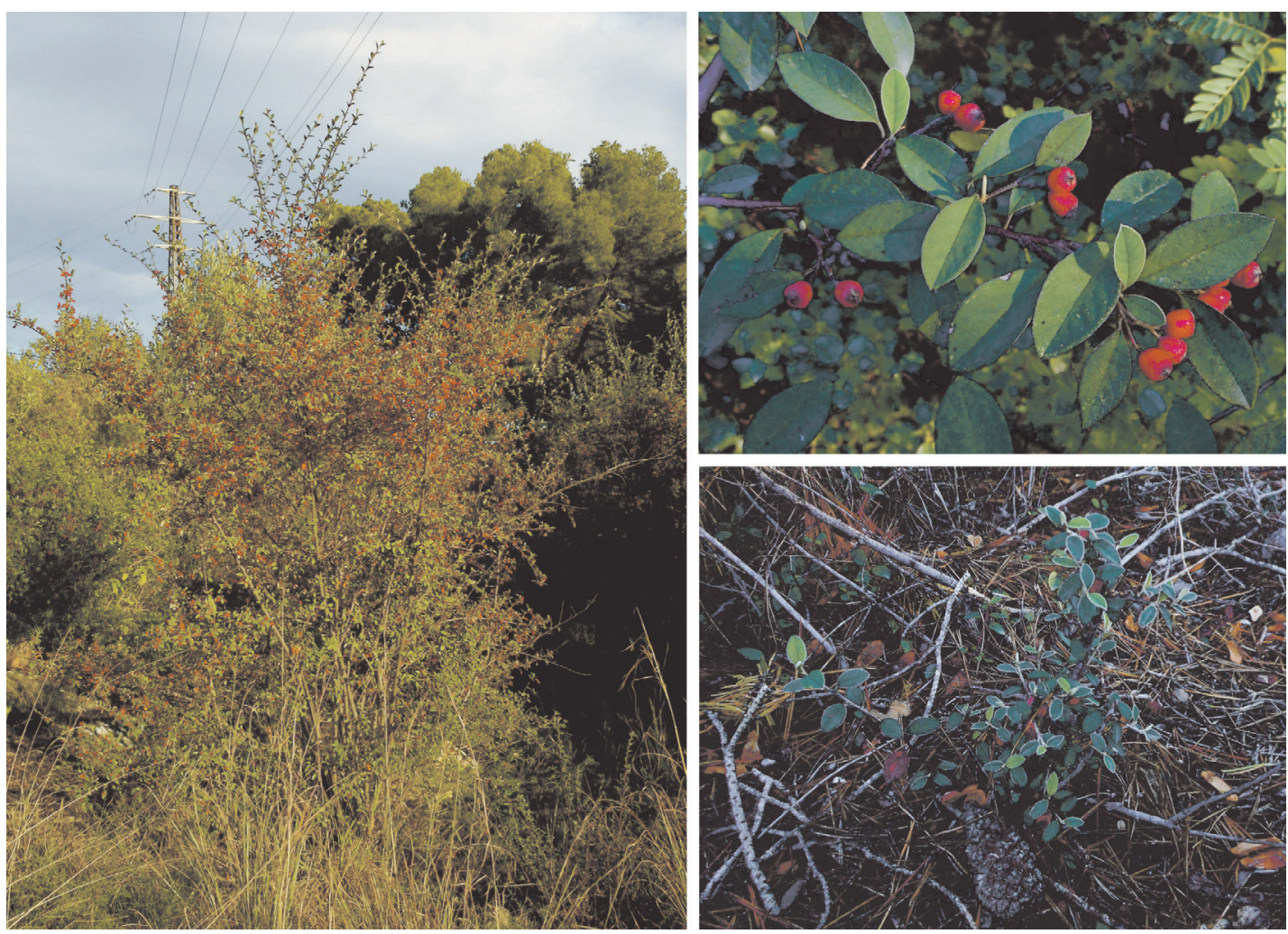

Fig. 2- Imágenes de la población de Cotoneaster pannosus de Tarragona. A la izquierda, individuo adulto; en la parte superior derecha, detalle de las hojas y los frutos; en la parte inferior derecha, individuo juvenil.

vo en plazas y jardines públicos pero también en jardines y fincas privadas; p. ej. de Andalucía, Aragón, CastillaLa Mancha, Extremadura y Galicia; http://www.gbif. org/species/3025572 y Guillot 2009, 2013). En la Comunidad Valenciana, C. pannosus ha sido citado en estado silvestre para las provincias de Castellón (en la comarca del Baix Maestrat; Royo 2006) y Valencia (en las comarcas de Camp de Túria, l'Horta Oest y Serrans; HerreroBorgoñón 2003, 2009; Herrero-Borgoñón et al. 2005). En Cataluña el arbusto lo citó por primera vez Casasayas (1989) en dos localidades (Viladrau y embalse de Sau) de la comarca de Osona (la primera situada dentro de la provincia de Girona y la segunda en la de Barcelona). Con posterioridad ha sido citado por Pyke (2008) en el área metropolitana de Barcelona (en la comarca del Baix Llobregat y en la ciudad de Barcelona) y por Aymerich (2013a, b) en hasta ocho localidades de la comarca del Berguedà, también en la provincia de Barcelona. Existe también un informe técnico donde se detalla la presencia de individuos asilvestrados de Cotoneaster cf. pannosus en la comarca barcelonesa del Vallès Oriental (Diputació de Barcelona 2009). Otro informe indica la presencia de C. pannosus en el Parc Natural del Montseny, sin especificar la localización exacta (Barber 2014); también existe constancia de su presencia en Parc Natural del Montgrí, les Illes Medes i el Baix Ter, donde se ha procedido a su erradicación junto a otras alóctonas (Diputació de Girona 2014).

Según apuntan Bossard \& Cuisance (1984), este arbusto se introdujo en Europa para su cultivo en jardinería a finales del siglo XIX (hacia el año 1889); los individuos de dos de las localidades recogidas por Aymerich (2013b) provendrían de unos jardines de un casa señorial que se crearon a principios del siglo XX, lo que indica que la introducción en nuestro país probablemente se produjo a caballo de los siglos XIX y XX. Su rápida capacidad de naturalización, que se manifiesta sobretodo en las zonas boscosas periurbanas, desaconseja su uso en jardinería; de hecho, localidades como Barcelona prohíben explícitamente su cultivo en ciertas áreas y proponen especies alternativas (Ajuntament de Barcelona 2014). 
J. López-Pujol y D. Guillot Ortiz Primera cita de Cotoneaster pannosus Franch. (Rosaceae) para la provincia de Tarragona...

Tabla 1

Lista y detalles de las localidades donde se ha reportado la presencia de Coroneaster pannosus en España.

* Véase Fig. 1. UTM no proporcionada por los autores pero deducida en base a los datos de las localidades

\begin{tabular}{|c|c|c|c|c|}
\hline $\mathbf{N}^{0 *}$ & UTM & Detalles de la localidad & Fuente & $\begin{array}{l}\mathrm{N}^{0} \text { herbario } \\
\text { (si existe) }\end{array}$ \\
\hline & Cataluña & & & \\
\hline 1 & $31 \mathrm{TCF} 5654$ & $\begin{array}{l}\text { Tarragona (Cala Romana), ca. } 20 \mathrm{~m} \text {, terreno } \\
\text { inculto, junto a la carretera N-340 }\end{array}$ & Presente trabajo & BC 941214 \\
\hline 2 & $31 \mathrm{TDF} 1386$ & Pallejà, claros de pinar, $220 \mathrm{~m}$ & Pyke (2008) & BC 810063 \\
\hline 3 & $31 \mathrm{TDF} 1484$ & $\begin{array}{l}\text { La Palma de Cervelló; seto bordeando campo, } \\
100 \mathrm{~m}\end{array}$ & Pyke (2008) & BC 906724 \\
\hline 4 & $31 \mathrm{TDF} 2785$ & Barcelona: Collserola, sobre Bonanova, $230 \mathrm{~m}$ & Pyke (2008) & BC 906723 \\
\hline 5 & $31 \mathrm{TDF} 3080$ & Barcelona: Montjuïc, descampado, $90 \mathrm{~m}$ & Pyke (2008) & - \\
\hline 6 & 31TDG0362 & $\begin{array}{l}\text { Berga, ladera sobre el margen derecho de la } \\
\text { Riera de Metge, } 840 \mathrm{~m} \text {; un individuo en un an- } \\
\text { tiguo bancal, a unos } 150 \mathrm{~m} \text { de un grupo de la } \\
\text { especie plantado como cercado vegetal en una } \\
\text { casa }\end{array}$ & $\begin{array}{l}\text { Aymerich } \\
(2013 b)\end{array}$ & - \\
\hline 7 & $\begin{array}{l}\text { 31TDG0561 y } \\
\text { 31TDG0661 }\end{array}$ & $\begin{array}{l}\text { Berga, urbanización Casa en Ponç, } 750-760 \text { m; } \\
\text { una decena de individuos reproductores dis- } \\
\text { persos en taludes y claros de garriga en el en- } \\
\text { torno del área urbanizada, hasta un máximo de } \\
150 \text { m de jardines }\end{array}$ & $\begin{array}{l}\text { Aymerich } \\
(2013 b)\end{array}$ & - \\
\hline 8 & 31TDG0658 & $\begin{array}{l}\text { Olvan, rodalia de la Colònia Rosal, } 490 \text { m, bar- } \\
\text { disses }\end{array}$ & $\begin{array}{l}\text { Aymerich } \\
\text { (2013a) }\end{array}$ & - \\
\hline 9 & $31 \mathrm{TDG} 0663$ & $\begin{array}{l}\text { Cercs, carretera de acceso a la presa de la } \\
\text { Baells, } 625 \mathrm{~m} \text {; } 2 \text { individuos en el sotobosque } \\
\text { de un encinar, a unos } 20 \mathrm{~m} \text { de un único ejem- } \\
\text { plar plantado como ornamental al lado de la } \\
\text { carretera }\end{array}$ & $\begin{array}{l}\text { Aymerich } \\
(2013 b)\end{array}$ & - \\
\hline 10 & 31TDG0744 & $\begin{array}{l}\text { Puig-reig, Colònia Vidal, } 380-390 \mathrm{~m} \text {; obser- } \\
\text { vados } 9 \text { individuos espontáneos en tres núcle- } \\
\text { os separados, en setos, zarzales y solares in- } \\
\text { cultos, hasta una distancia de } 180 \mathrm{~m} \text { de los } \\
\text { aparentes «individuos-fuente» plantados en el } \\
\text { jardín de la «torre» de esta colonia }\end{array}$ & $\begin{array}{l}\text { Aymerich } \\
\text { (2013b) }\end{array}$ & - \\
\hline 11 & 31TDG1065 & $\begin{array}{l}\text { Vilada, bajo la Coromina, } 690 \mathrm{~m} \text {; un individuo } \\
\text { en el sotobosque de un pinar joven de Pinus } \\
\text { sylvestris, a } 50 \mathrm{~m} \text { de una casa }\end{array}$ & $\begin{array}{l}\text { Aymerich } \\
(2013 b)\end{array}$ & - \\
\hline 12 & 31TDG1165 & $\begin{array}{l}\text { Vilada, solana sobre el pueblo, } 760-790 \mathrm{~m} \text {; } \\
\text { unos } 100 \text { individuos de todos los tamaños dis- } \\
\text { persos en una superficie de } 0,9 \text { ha, en el soto- } \\
\text { bosque de una repoblación de pinos y en setos, } \\
\text { hasta distancias de } 150 \mathrm{~m} \text { de jardines }\end{array}$ & $\begin{array}{l}\text { Aymerich } \\
\text { (2013b) }\end{array}$ & - \\
\hline
\end{tabular}


Tabla 1 (cont.)

\begin{tabular}{|c|c|c|c|c|}
\hline $\mathrm{N}^{0 *}$ & $\mathrm{UTM}^{* *}$ & Detalles de la localidad & Fuente & $\begin{array}{l}\mathrm{N}^{0} \text { herbario } \\
\text { (si existe) }\end{array}$ \\
\hline 13 & 31TDG1579 & $\begin{array}{l}\text { Castellar de n'Hug, Clot del Moro, entorno de } \\
\text { la antigua fábrica de cemento Asland, 940-995 } \\
\text { m; muy abundante en los espacios antrópicos } \\
\text { abandonados (centenares de individuos, quizá } \\
\text { unos pocos miles) y escaso ( } 30-50 \text { individuos } \\
\text { observados) en hábitats naturales adyacentes } \\
\text { (laderas rocosas y bosques abiertos); este im- } \\
\text { portante núcleo tendría su origen en los jardi- } \\
\text { nes de la «torre» de esta antigua fábrica, cre- } \\
\text { ados a principios del siglo XX, y se han } \\
\text { observado individuos espontáneos hasta } 170 \\
\text { m de distancia }\end{array}$ & $\begin{array}{l}\text { Aymerich } \\
(2013 b)\end{array}$ & - \\
\hline 14 & $31 \mathrm{TDG} 32 * *$ & $\begin{array}{l}\text { Diversos exemplars del que sembla ser Coto- } \\
\text { neaster pannosus estan colonitzant el sotabosc } \\
\text { de l'alzinar i la roureda de l'entorn d'Aigua- } \\
\text { freda i la vall de Martinet }\end{array}$ & $\begin{array}{l}\text { Diputació de } \\
\text { Barcelona } \\
\text { (2009) }\end{array}$ & - \\
\hline 15 & $\begin{array}{l}31 \mathrm{TDG} 42 \mathrm{y} / 0 \\
31 \mathrm{TDG} 52\end{array}$ & Parc Natural del Montseny & Barber (2014) & - \\
\hline 16 & $31 \mathrm{TDG} 43$ & Parets i marges de carrers de Viladrau & $\begin{array}{l}\text { Casasayas } \\
(1989)\end{array}$ & - \\
\hline 17 & $31 \mathrm{TDG} 54$ & Prop de la presa de Sau & $\begin{array}{l}\text { Casasayas } \\
(1989)\end{array}$ & - \\
\hline \multirow[t]{2}{*}{18} & $31 \mathrm{TEG} 16^{* *}$ & $\begin{array}{l}\text { Paraje de las Planasses - Salpatx (término mu- } \\
\text { nicipal de l'Escala, dentro de los límites del P. } \\
\text { Nat. del Montgrí, les Illes Medes i el Baix Ter) }\end{array}$ & $\begin{array}{l}\text { Diputació de } \\
\text { Girona (2014) }\end{array}$ & - \\
\hline & $\begin{array}{l}\text { Comunidad } \\
\text { Valenciana }\end{array}$ & & & \\
\hline 19 & 30SXK5812 & Valencia: Titaguas, La Tosquilla, $586 \mathrm{~m}$ & $\begin{array}{l}\text { Herrero-Borgo- } \\
\text { ñón (2009) }\end{array}$ & VAL 193248 \\
\hline 20 & 30SYJ1666 & $\begin{array}{l}\text { Valencia: Torrente, El Vedat, } 120 \mathrm{~m} \text {, naturali- } \\
\text { zado en pinar de } P \text {. halepensis }\end{array}$ & $\begin{array}{l}\text { Herrero-Borgo- } \\
\text { ñón (2003) }\end{array}$ & VAL 144933 \\
\hline 21 & 30SYJ2096 & $\begin{array}{l}\text { Valencia: Serra, pr. senda al Puntal, } 360 \mathrm{~m} \text {, en } \\
\text { campos abandonados }\end{array}$ & $\begin{array}{l}\text { Herrero-Borgo- } \\
\text { ñón et al. (2005) }\end{array}$ & VAL 146107 \\
\hline 22 & 31TBE77 & $\begin{array}{l}\text { Marjal de Peníscola, rere el càmping, herbas- } \\
\text { sar ruderal humit, } 1 \mathrm{~m}\end{array}$ & Royo (2006) & BCN 21712 \\
\hline
\end{tabular}

\section{BIBLIOGRAFÍA}

AJunTAMENT DE BARCELONA 2014. Estudi d'espècies invasores a la ciutat de Barcelona i proposta d'espècies alternatives. Ajuntament de Barcelona, Barcelona.
ANDREU, J. \& PinO, J. 2013. El projecte EXOCAT. Informe 2013. Departament d'Agricultura, Ramaderia, Pesca, Alimentació i Medi Natural (Generalitat de Catalunya), Barcelona. 
Andreu, J. \& Vilà, M. 2010. Risk analysis of potential invasive plants in Spain. J. Nat. Conserv. 18: 34-44.

ANónimo 2014. Silverlead cotoneaster - Cotoneaster pannosus Franch. Invasive plant atlas of the United States. Consultado el 15/11/2014, en http://www.invasiveplantatlas.org/subject.html?sub=5377

Aymerich, P. 2013a. Contribució al coneixement florístic del territori ausosegàrric (NE de la península Ibèrica). Orsis 27: 209-259.

AYMerich, P. 2013b. Plantas alóctonas de origen ornamental en la cuenca alta del río Llobregat (Cataluña, noreste de la Península Ibérica). Bouteloua 16: 52-79.

BAKER, M. L. 2005. Contributions to a catalogue of alien plants in Tasmania I. Pap. Proc. R. Soc. Tasman. 139: 33-48.

Barber, J. (Dir.). 2014. Pla de conservació del Parc Natural del Montseny. Reserva de la Biosfera. Diputació de Barcelona, Barcelona.

Blanca, G. 1998. Cotoneaster. En: F. Muñoz \& C. Navarro (Eds.), Flora iberica, 6: 394-401. Real Jardín Botánico (CSIC), Madrid.

Bossard, R \& Cuisance, P. 1984. Arbres et arbustes d'ornement des régions tempérées et méditerranéennes. Tecnique $\&$ Documentation-Lavoisier, Paris.

Cal-IPC (California Invasive Plant Council) 2006. California invasive plant inventory. California Invasive Plant Council, Berkeley, CA.

Camelo, L.; Díaz-Espinosa, A. \& Díaz, J. E. 2012. Cotoneaster pannosus. En A. M. Díaz-Espinosa, J. E. Díaz-Triana \& O. Vargas (Eds.), Catálogo de plantas invasoras de los humedales de Bogotá: 155-158. Grupo de Restauración Ecológica de la Universidad Nacional de Colombia y Secretaría Distrital de Ambiente, Bogotá, DC.

CASAsayas, T. 1989. La flora al lòctona de Catalunya. Catàleg raonat de les plantes vasculars exòtiques que creixen sense cultiu al NE de la Península Ibèrica. Tesis Doctoral. Facultat de Biologia, Universitat de Barcelona.

Celesti-Grapow, L.; Alessandrini, A.; Arrigoni, P. V. et al., 2009. Inventory of the non-native flora of Italy. Plant Biosyst. 143: 386-430.

DAISIE (DELIVERING ALIEN INVASIVE SPECIEs INVENTORIES For Europe) 2014. Cotoneaster pannosus - Species Factsheet. DAISIE project website. Consultado el 14/11/2014, en http://www.europe-aliens.org/speciesFactsheet.do?speciesId $=13306 \#$

Diputació DE BARCELONA 2009. Agenda 21 supramunicipal. Aiguafreda, el Brull, Cànoves i Samalús, Montseny, Sant Marti de Centelles i Tagamanent-Document I: Memòria descriptiva. Diputació de Barcelona, Barcelona.

Diputació DE GIRONA 2014. Actuacions i Projectes 2012 - Eradicació de la flora exòtica invasora al paratge de la Planassa-Salpatx (l'Escala). Diputació de Girona, Girona. Consultado el 18/11/2014, en http://www.ddgi.cat/lportal/ web/medi-ambient/actuacions-1\%60projectes-2012

FAnG, J.; Wang, Z.\& TANG, Z. (EDS.) 2011. Atlas of woody plants in China - Distribution and climate, 1. Higher Education Press, Beijing and Springer-Verlag Berlin/Heidelberg.
Fraga, P. \& Del Toro, V. 2010. La jardineria i les plantes invasores: alternatives i eines pràctiques per a la seva gestió. En: C. Álvarez (Ed.), Seminari sobre espècies intruduides i invasores a les Illes Balears: 153-174. Conselleria de Medi Ambient i Mobilitat (Govern de les Illes Balears), Sóller.

Fryer, J. \& Hylmö, B. 2009. Cotoneasters - A comprehensive guide to shrubs for flowers, fruit, and foliage. Timber Press, Portland \& London.

Guillot, D. 2009. Plantas ornamentales de Noguera (Teruel) (II). Angiospermas dicotiledóneas. Rehalda 11: 67-83.

Guillot, D. 2013. Plantas ornamentales de Molina de Aragón (Guadalajara, España). Bouteloua 14: 64-114.

Heenan P. B.; Lange P. J. de; Cameron E. K.; Ogle C. C. \& Champion P. D. 2004. Checklist of dicotyledons, gymnosperms, and pteridophytes naturalised or casual in New Zealand: additional records 2001-2003. N. Z. J. Bot. 42: 797-814.

Herrero-Borgoñón, J. J. 2003. Dos Cotoneaster (Rosaceae) de uso ornamental naturalizados en Valencia. Flora Montiberica 24: 3-5.

Herrero-BorgoÑón, J. J. 2009. Notas sobre flora alóctona valenciana. Flora Montiberica 43: 19-25.

Herrero-Borgoñón, J. J.; Ferrer, P. P. \& Guara, M. 2005. Notas sobre la flora alóctona valenciana de origen ornamental. Acta Bot. Malacitana 30: 182-187.

Hyde, M. A.; Wursten, B. T.; Ballings, P. \& Coates PalgraVE, M. 2014. Cotoneaster pannosus Franch. Flora of Zimbabwe. Consultado el 17/11/2014, en http://www.zimbabweflora.co.zw/speciesdata/species.php?species_id=125330

InBUy (BASE DE DATOS DE INVASIONES BIOLÓGICAS PARA URUGUAY) 2011. Cotoneaster pannosus Franch. Facultad de Ciencias, Universidad de la República. Montevideo. Consultado el 14/11/2014, en http://inbuy.fcien.edu.uy/ fichas_de_especies/DATAonline/DBASEimpresiones/ Cotoneaster_pannosus_i.pdf

Lorence, D. H.; Flynn, T. W. \& Wagner, W. L. 1995. Contributions to the flora of Hawai'i. III. New additions, range extensions, and rediscoveries of flowering plants. Bish. Mus. Occas. Pap. 41: 19-58.

Lu, L. \& Brach, A. R. 2003. Cotoneaster. En Z. Y. Wu, P. H. Raven \& D. Y. Hong (Eds.), Flora of China, 9 (Pittosporaceae through Connaraceae): 85-108. Science Press, Beijing \& Missouri Botanical Garden Press, St. Louis.

Macdonald, I. A. W.; Reaser, J. K.; Bright, C.; Neville, L. E.; Howard, G. W.; Murphy, S. J. \& Preston, G. (Eds.). 2003. Invasive alien species in southern Africa: national reports \& directory of resources. The Global Invasive Species Programme, Cape Town, South Africa.

NNSS (Non-Native Species SeCretariat) 2014. Silverleaf Cotoneaster, Cotoneaster pannosus. Great Britain Non-native Species Secretariat, Animal Health and Veterinary Laboratories Agency, Sand Hutton (UK). Consultado el 16/11/2014, en http://www.nonnativespecies.org/ $\mathrm{f}$ actsheet/factsheet.cfm? speciesId $=985$

PyKe, S. 2008. Contribución al conocimiento de la flora alóctona catalana. Collect. Bot. 27: 95-104. 
RoYo, F. 2006. Flora i vegetació de les planes i serres litorals compreses entre el riu Ebro i la serra d'Irta. Tesis Doctoral. Facultat de Biologia, Universitat de Barcelona.

QuEENSLAND GOVERNMENT 2011. Silver-leaved cotoneaster-Cotoneaster pannosus. Queensland Government, Brisbane. Consultado el 17/11/2014, en http://keyserver.lucidcentral. org/weeds/data/03030800-0b0 7-490a-8d040605030c0f01/media/Html/Cotoneaster_pannosus.htm

Sanz, M.; Dana, E. D. \& Sobrino, E. 2004. Atlas de las plantas alóctonas invasoras en España. Dirección General para la Biodiversidad, Ministerio de Medio Ambiente. Madrid.

SAnZ, M.; Guillot, D. \& Del Toro, V. 2011. La flora alóctona de la Comunidad Valenciana (España). Bot. Complut. 35: 97-130.
Sanz, M.; González, F. \& Serreta, A. 2009. La flora alóctona de Aragón (España). Bot. Complut. 33: 69-88.

SAPD (Swaziland's Alien Plants Database) 2014. Cotoneaster pannosus Franch. Swaziland National Trust Commission, Lobamba, Swaziland. Consultado el 16/11/2014, en http://www.sntc.org.sz/alienplants/speciesinfo.asp?spid= 343

Valkenburg, J. van; Brunel, S.; Brundu, G.; Ehret, P.; FoLLAK, S. \& ULUDAG, A. 2014. Is terrestrial plant import from East Asia into countries in the EPPO region a potential pathway for new emerging invasive alien plants? Bulletin OEPP/EPPO Bulletin 44: 195-204.

VIEIRA, R. M. S. 2002. Flora da Madeira - plantas naturalizadas no Arquipélago da Madeira. Bol. Mus. Munic. Funchal (Hist. Nat.) Suppl. 8: 5-281. 
